\title{
Condiciones de la gestión del talento humano que favorecen el desarrollo de capacidades dinámicas
}

\author{
Karelis del C. Barrios-Hernández*, Enohemit Olivero-Vega y Brenda Figueroa-Saumet \\ Facultad de Administración y Negocios, Universidad Simón Bolívar, Barranquilla-Colombia. \\ (correo-e: kbarrios22@unisimonbolivar.edu.co; eolivero@unisimonbolivar.edu.co; brenda.figueroa@unisimon.edu.co) \\ * Autor a quien debe ser dirigida la correspondencia
}

Recibido Ago. 2, 2019; Aceptado Sep. 26, 2019; Versión final Dic. 17, 2019, Publicado Abr. 2020

\begin{abstract}
Resumen
Se estudia y analiza las condiciones de la gestión del talento humano que favorecen el desarrollo de capacidades dinámicas de un grupo de directivos de empresas. Se planteó una investigación cuantitativa, mediante la aplicación de una encuesta compuesta por catorce ítems medidos en una escala tipo Likert, dirigida a setenta líderes ubicados en empresas de diferentes sectores de la economía en la ciudad de Barranquilla, Colombia. Los resultados muestran que el espacio compartido de conocimiento y el estilo de dirección de liderazgo que promueva la transformación y el cambio tienen un impacto positivo en el desarrollo de capacidades dinámicas de los líderes, para adaptarse a las condiciones cambiantes de entorno.
\end{abstract}

Palabras clave: capacidades dinámicas; gestión del talento humano; estilo de dirección.

\section{Conditions of human talent management that favor the development of dynamic capabilities}

\begin{abstract}
The conditions of human talent management that favor the development of dynamic capabilities of a group of business executives are studied and analyzed. Quantitative research was proposed through the application of a survey composed of fourteen items measured on a Likert scale, addressed to seventy leaders of companies from different sectors of the economy, located in the city of Barranquilla, Colombia. The results show that the shared knowledge space and a leadership style that promotes transformation and changes have positive impact on the development of the dynamic capabilities of the leaders, to adapt themselves to the changing corporate environmental conditions.
\end{abstract}




\section{INTRODUCCIÓN}

En el ámbito empresarial se ha reconocido recientemente el papel estratégico que juega el talento humano en la creación de valor de las organizaciones, al tiempo que los cambios del entorno, están obligando a trasformar la realidad empresarial, poniendo de relieve la importancia de la gestión del talento humano, como la fuente principal de adaptación al entorno. La forma como cada organización asume esos cambios, depende de la rapidez de adaptación, la generación de ideas novedosas y el desarrollo de nuevas rutinas organizacionales que propicien nuevos procesos para responder a la turbulencia de ese entorno (BarriosHernández et ál., 2017). En un mundo donde los mercados, productos, tecnología, competidores cambian de manera vertiginosa, la gestión del talento humano en las empresas se convierte en un elemento clave de su éxito. Por tanto, los procesos mediante los cuales se crea y se gestiona el conocimiento y por ende el talento humano, constituyen la fuente de capacidades dinámicas y de ventaja competitiva sostenible (Acosta y Fischer, 2013). No obstante, hay que tener en cuenta que la gestión del talento humano se materializa en aspectos cuyo gobierno facilita que el talento humano se convierta en una fuente de capacidad dinámica para la organización (Acosta, 2010; Acosta y Fischer, 2013) y en la capacidad de responder al dinamísmo del entorno (Teece, 2009; Acosta y Fischer, 2013).

En este sentido uno de los factores que inciden en la dinámica de las organizaciones son las personas. Es por eso, que la gestión del talento humano se ha convertido en un pilar estratégico de la gestión empresarial moderna. Una de las fases claves de la gestión del talento humano, es la dirección del conocimiento. Bierly y Chakrabarti (1996), afirman que la capacidad de obtener conocimiento puede ser considerada la única ventaja competitiva sostenible de la empresa. Dicha ventaja deriva de la adquisición, desarrollo, transferencia y obtención de nuevos conocimientos en la organización, que deben ser materializados en la generación de innovaciones, como resultados superiores que permitan a la empresa mejorar su posición competitiva (Acosta, 2010). Dentro de este proceso, las capacidades dinámicas y el talento humano juegan un papel muy importante, porque permiten explotar las oportunidades que ofrece el entorno, mediante la creación de productos nuevos o mejorados.

El fundamento de las capacidades dinámicas se encuentra en el enfoque de recursos y capacidades. Por una parte están los recursos, Penrose (1959), señala que el recurso más importante de una firma es la gerencia. Para Barney (1991), los recursos son heterogéneos e inmóviles entre las empresas, y propone que para que estos puedan tener el potencial de generar ventaja competitiva, deben ser: valiosos, raros, imperfectamente imitables e insustituibles. Por otra parte están las capacidades las cuales se pueden concebir como la construcción de la sabiduría colectiva en cualquier organización (Garzón, 2013), y nacen a lo largo del tiempo mediante las interacciones que surgen entre los recursos de la empresa (Venture, 1994). Es así como, una organización posee una capacidad cuando hace algo que es apreciado, y lo hace de manera efectiva, comparado con otras organizaciones menos capaces de obtener una solución. (Dávila, 2013; Barrios y Olivero 2015).

Existen tres niveles de jerarquía de capacidades, en primer nivel se sitúan los recursos, en el nivel siguiente las capacidades y en el último nivel las capacidades dinámicas, las cuales contribuyen al desarrollo de ventaja competitiva sostenible en las organizaciones en el largo plazo (Barrios y Olivero, 2015; Aguilar y Yepes, 2006; Winter, 2003; Garzón, 2013). Barrios y Olivero (2015), manifiestan que para entender cómo se crean ventajas competitivas sostenibles en entornos turbulentos, la literatura (Barney, 1991; Teece, 2007; Teece, 2009) ha demostrado que esto es posible a través del desarrollo de las capacidades dinámicas, las cuales permiten a la empresa reconfigurar su base de recursos y adaptarse a las condiciones cambiantes del mercado, con el objetivo de lograr una ventaja competitiva (Zahra y George, 2002). Para Mendoza (2013) las capacidades dinámicas se ubican entre lo interno y lo externo, es decir en lo interno están los recursos y capacidades de la organización y en lo externo, el entorno para la adaptación a las condiciones cambiantes del entorno (Barrios y Olivero, 2015).

\section{OTROS ANTECEDENTES}

Hya tras concetos que es ocnveniente revisar para el trtabajo que se dearrolla y se presneta en este articulo: las capacidades dinámicas, la gestión del talento humano, y las condiciones de la gestión del talento humano

\section{Capacidades dinámicas}

Acosta et al., (2013) argumentan que las capacidades dinámicas consisten en procesos estratégicos y organizacionales específicos (como desarrollo de productos, alianzas, o toma de decisiones estratégicas), en empresas que operan en mercados dinámicos mediante la manipulación de recursos hacia nuevas estrategias de creación de valor. El enfoque de estos autores establece que aquellas capacidades que favorecen la adaptación y desarrollo de las empresas, responden a un determinado conjunto de criterios que explican su 
valor y escasez, fruto de la heterogeneidad entre empresas y de su imperfecta movilidad, y son, al mismo tiempo, insustituibles, difíciles de imitar e intransferibles (Barney, 1991). La tabla 1 expone las principales aportaciones sobre capacidades dinámicas. Es así como las capacidades dinámicas suponen un despliegue de capacidades insustituibles, intransferibles y difíciles de imitar como proceso estratégico de conformación, reconformación, configuración, reconfiguración y protección de la base de recursos y capacidades esenciales de una empresa, para la adaptación a las condiciones cambiantes del entorno y la generación de ventaja competitiva sostenible (Barrios y Olivero, 2015).

Tabla 1. Principales aportaciones sobre capacidades dinámicas. Fuente: (Barrios y Olivero, 2015)

\begin{tabular}{|c|c|}
\hline Autor & Definiciones Principales \\
\hline Teece y Pisano (1994) & $\begin{array}{l}\text { "Subconjunto de competencias o capacidades que permiten a la empresa } \\
\text { crear nuevos productos y procesos, respondiendo así a circunstancias } \\
\text { cambiantes del mercado"(p.541) }\end{array}$ \\
\hline Helfat (1997) & $\begin{array}{l}\text { "Las capacidades dinámicas permiten a las empresas crear nuevos productos } \\
\text { y procesos y responder a las condiciones cambiantes del mercado" (p.339) }\end{array}$ \\
\hline Zahra y George, (2002) & $\begin{array}{l}\text { "Permiten a la empresa reconfigurar su base de recursos y adaptarse a las } \\
\text { condiciones cambiantes del mercado con el objetivo de lograr una ventaja } \\
\text { competitiva" (p.185) }\end{array}$ \\
\hline Helfat y Peteraf (2003) & $\begin{array}{l}\text { "Por definición, las capacidades dinámicas implican adaptación y cambio, } \\
\text { porque construyen, integran o reconfiguran otros recursos o capacidades" (p. } \\
997)\end{array}$ \\
\hline Augier y Teece (2007) & $\begin{array}{l}\text { "Capacidad (inimitable) con la que cuenta la empresa para formar, reformar, } \\
\text { configurar y reconfigurar su base de activos para poder responder a los } \\
\text { cambios en mercados y tecnologías" (p.179) }\end{array}$ \\
\hline Wang y Ahmed (2007) & $\begin{array}{l}\text { "Orientación en el comportamiento de la empresa a la continua integración, } \\
\text { reconfiguración, renovación y recreación de sus recursos y capacidades y, } \\
\text { más importante, al incremento y reconstrucción de sus capacidades } \\
\text { esenciales en respuesta a un entorno cambiante para conseguir mantener la } \\
\text { ventaja competitiva" (p. 35) }\end{array}$ \\
\hline Eisenhardt y Martin (2000) & $\begin{array}{l}\text { "Consisten en procesos estratégicos y organizativos específicos (como } \\
\text { desarrollo de productos, alianzas, o toma de decisiones estratégicas) que } \\
\text { crean valor en empresas que operan en mercados dinámicos mediante la } \\
\text { manipulación de recursos hacia nuevas estrategias de creación de valor" (p. } \\
1106 \text { ) }\end{array}$ \\
\hline Teece (2009) & $\begin{array}{l}\text { "La particular (inimitable) capacidad que poseen las empresas de negocio } \\
\text { para conformar, reconformar, configurar y reconfigurar los activos, de tal } \\
\text { manera que puedan responder a los mercados y tecnologías cambiantes y } \\
\text { escapar del beneficio cero" ( } p .94 \text { ) }\end{array}$ \\
\hline
\end{tabular}

La funcionalidad de la capacidad dinámica descansa en la posibilidad de reconfigurar los recursos y capacidades, y aunque estas se relacionan con la respuesta al entono, no toda respuesta puede considerarse capacidad dinámica, por eso, existen diferentes jerarquías acorde a los diferentes estudiosos del tema. Para Teece (2009), las capacidades dinámicas pueden ser: Capacidad de aprendizaje organizacional, la innovación, el diseño de modelo de negocio, decisiones heurísticas de asignación de recursos, orquestación (adaptación, reempleo y reconfiguración) de activos, negociación y transacción, gobierno y alineación. Mientras para Eisenhardt y Martin (2000) las capacidades dinámicas se ven representadas en los Equipos de I+D transfuncionales, rutinas de desarrollo de nuevos productos, rutinas de control de calidad y rutinas de transferencia de conocimiento y tecnología. Para autores como Wang y Ahmed (2007) y Garzón (2013) la capacidad de absorción, capacidad de innovación, capacidad de aprendizaje y capacidad de adaptación. En este sentido, las capacidades dinámicas requieren del talento humano para el desarrollo de las empresas que logran éxito en un ambiente competitivo, debido a que permiten la generación de respuestas rapidas y flexible al entorno y la optimización del curso estratégico global del futuro de la empresa.

\section{Gestión del talento humano}

En la era actual del conocimiento y la información, el factor estratégico de competitividad sostenible de las organizaciones es el talento humano. Los conocimientos de las personas son la clave de la empresa y explican buena parte de la valoración que el mercado concede a una organización (Mejía y Jaramillo, 2006). En otras 
palabras, significa que lo más importante y estratégico para el desarrollo de una empresa es utilizar el talento humano como factor de generación de conocimiento productivo; finalmente este conocimiento se constituye en la base de la innovación y la productividad (Nonaka y Takeuchi, 1995) y, además, en fuente directa de la ventaja competitiva sostenible (Mejía y Jaramillo, 2006).

De esta forma, el objetivo principal de una empresa debe ser potenciar el desempeño del trabajador, tratando de que su acción tenga efectos en toda la cadena productiva (Mejía et al., 2013). Por tanto, la gestión del talento humano tiene la oportunidad de ser protagonista en los procesos de transformación organizacional, ya que estos hacen posible que sea el colectivo de los miembros de la organización quienes propicien los cambios que permitirán mejorar los resultados de la organización, para responder a la dinámica del mercado (Calderon et al., 2009).

Por eso, la gestión del talento humano debe mantener una visión de futuro, para anticipar los cambios y evaluar las consecuencias de ellos, de modo que pueda retransmitir esta visión a todas las personas que gestiona y desempeñar un papel importante como facilitador de la innovación, lo cual puede lograr por una parte a través del liderazgo transformacional, para permitirle a los empleados descubrir y emplear el conocimiento, animando a su vez el trabajo en equipo para promover la co-innovación, de modo que se desarrolle el aprendizaje a través de conocimientos multidisciplinarios (Laursen, 2002). Por tanto, el talento humano no puede desarrollarse de manera espontánea para la generación de capacidades dinámicas, se necesita de un espacio para la generación de ideas ampliamente conocido en la literatura como espacio compartido de conocimiento: Ba (Acosta, 2010). Este concepto fue inicialmente mencionado por Nishida (1921) y posteriormente desarrollado por Shimizu (1995). Por tanto, hace referencia al ambiente en el cual el conocimiento es compartido, creado y utilizado, de tal manera que se pueda potenciar el talento del trabajador para responder a las condiciones cambiantes del entorno, independientemente del lugar que ocupe dentro de la jerarquía laboral (Mejía y Jaramillo, 2006).

\section{Condiciones de la gestión del talento humano}

Es así como la gestión del talento humano juega un papel preponderante en el desarrollo de capacidades dinámicas. De acuerdo a la literatura (Bravo y Herrera, 2009) estas capacidades requieren de la gestión del talento humano a través de procesos dinámicos, que permiten a la empresa adaptar la base de recursos y las habilidades empresariales para afrontar los cambios del entorno y generar nuevas formas de generación de ventajas competitivas (Teece et al., 1997; Eisenhardt y Martín, 2000; Acosta, Longo-Samoza, y Fischer, 2013). Así mismo, para que la gestión del talento humano propicie el desarrollo de capacidades dinámicas se requiere de la creación de conocimiento (Nonaka y Takeuchi, 1995), la absorción de conocimiento (Zahra y George, 2002), la integración de conocimiento (Grant 1996) y la reconfiguración de conocimiento (Lavie, 2006).

La creación y la absorción del conocimiento, indica que el talento humano se gestiona en la medida que los individuos que conforman la organización permanezcan en contacto permanente con su entorno, de tal manera, que la percepción organizacional se oriente hacia el medio (Barrios y Olivero, 2015). Por un lado, analiza y busca señales sobre innovaciones potenciales, amenazas y oportunidades de cambio e identifica aquellos factores 0 variables que constituyen oportunidades para la empresa, con el propósito de tomar ventaja de ellas y aprovecharlas para el desarrollo de la organización y, por otro lado, contrarrestar las posibles amenazas que enfrenta o deberá enfrentar la organización, con el fin de neutralizar y minimizar su impacto negativo. Estas oportunidades y amenazas pueden presentarse en el contexto jurídico o normativo, económico, social, político, ambiental y cultural (Barrios y Olivero, 2015).

Por su parte la absorción del conocimiento se constituye como la habilidad y motivación de los empleados para obtener conocimiento externo y utilizarlo. Sin embargo, para gestionar el talento humano, es necesario integrar el conocimiento de los individuos y de la organización, es decir se requiere de una previa interacción y colaboración entre las personas y el medio (Hung, Kao, y Chu, 2008). La interacción enfatiza la utilización e intercambio de información entre unidades funcionales. La colaboración se fundamenta en el trabajo colectivo entre departamentos o entre organizaciones facilitando la capacidad de relacionamiento (Grant, 1996; Barrios y Olivero, 2015). Por eso las capacidades internas existentes y su interacción con fuentes de conocimiento externo pueden afectar positivamente el nivel de innovación de las compañías Bravo y Herrera, 2009).

Es así como, las empresas en la medida que incorporen la gestión del conocimiento pueden innovar mediante el manejo direccionado de los procesos de identificar, compartir, generar, retener y aplicar conocimiento, formando al capital humano (Hernández et al., 2014). Es así como la organización gestiona su talento humano, en la medida que establezca relaciones de cooperación tanto externa como interna. Para Becerra y Álvarez, (2011) la cooperación se manifiesta por la capacidad que tienen las empresas para constituir grupos 
o redes de conocimiento. De esta manera, las personas pueden reconocer las tendencias, potencialidades y rupturas que afectaran el desarrollo futuro de la empresa, por lo que se requiere de su conocimiento y habilidades para captar o desarrollar nuevas ideas y explorar el medio y a su vez establecer contacto efectivo y relaciones de colaboración con los actores del entorno (Mendoza, 2013; Barrios y Olivero, 2015).

Por un lado, la captación y desarrollo de ideas novedosas implica que la gestión del talento humano fomenta la transformación y el cambio. Esto implica garantizar un espacio de interacción entre las personas, el trabajo y el contexto de la empresa o institución. Por tanto, gestionar a la persona significa comprender su talento y alinearlo a los objetivos organizacionales para promover la innovación. Asimismo, a mayor número de ideas creativas generadas, mayor será el número de opciones disponibles para su implantación a la hora de innovar y, por ende, existirán más posibilidades de generación de nuevo conocimiento y de combinaciones del existente, y mayor será la flexibilidad de la organización para responder a las demandas y oportunidades externas desarrollando capacidades organizacionales dinámicas (Barrios-Hernández et ál., 2017). Pero el ba no es solo el resultado de un espacio compartido -físico, virtual y mental- sino también la expresión de un conjunto de roles y habilidades de los directivos que permite una gestión eficiente del conocimiento. En este caso, el liderazgo articula la visión y anima a los miembros de la organización a que se involucren activamente en proyectos innovadores (Acosta, 2010). Gracias a las habilidades del líder se puede trasformar y promover el cambio mediante la gestión del talento, a fin que este proceso creativo sea útil a la organización y favorezca el desarrollo de la innovación. La responsabilidad más grande que tiene el directivo es ayudar a los individuos a aprender. Es ahí donde reside la base esencial de su liderazgo. El líder no necesita conocer todas las respuestas sino entender cuestiones clave como los límites y el potencial del conocimiento, los recursos técnicos, organizativos y económicos que requiere su desarrollo, la dirección y la velocidad del cambio y su riesgo (Acosta, 2010).

Las hipótesis de investigación para contrastar empíricamente los resultados, son dos: H1. Las capacidades dinámicas se desarrollan en la medida que la organización aproveche las oportunidades y relaciones con su entorno; y H2 Las condiciones facilitadoras de la gestión del talento humano que favorecen el desarrollo de capacidades dinámicas son el desarrollo de un espacio de interacción de conocimiento entre las personas y un estilo de liderazgo que promueva la transformación y el cambio.

\section{METODOLOGÍA}

Teniendo en cuenta que este articulo tiene por objetivo describir las condiciones de la gestión del talento humano que favorecen el desarrollo de capacidades dinámicas, la investigación desarrollada fue de tipo descriptiva y exploratoria puesto que permitió aplicar un conjunto de métodos y procedimientos para recolectar datos puros, y generar estructuras de datos que describen las condiciones de la gestión $\square$ del talento humano que favorecen el desarrollo de capacidades dinámicas. La población seleccionada para la investigación fueron 70 directivos de empresas de diferentes sectores ubicadas en la ciudad de Barranquilla. Se consideró apropiado realizar una investigación cuantitativa. La técnica utilizada para la recolección de datos fue la encuesta, mediante la elaboración de un cuestionario compuesto por 14 ítems distribuidos en dos variables: gestión del talento humano y capacidades dinámicas. Estas variables fueron medidas con una escala tipo Likert en el cuestionario (siempre, casi siempre, algunas veces, casi nunca y nunca).

En el diseño del Instrumento se cumplieron varias fases, en primer lugar, se elaboró un banco de ítems o preguntas, con posibilidades a ser utilizados en el instrumento final a aplicar. En segundo lugar, se realizaron los filtros pertinentes, para finalmente obtener una en los datos se envió el cuestionario a tres expertos, quienes solicitaron ajustes en la claridad y brevedad. Para ser operativo el instrumento, se desarrolló en utilizando la escala tipo Likert de 5 puntos que expresaban el grado de importancia (de menos a más) con la afirmación que se manifestaba en cada pregunta ( $1=$ Nunca; $2=$ Casi Nunca; 3=Algunas Veces; 4= Casi Siempre; $5=$ Siempre), En tercer lugar y con el propósito de determinar la validez y evitar sesgos Logrando un cuestionario final de 14 ítems validados, a los cuales se les realizó el respectivo análisis de la confiabilidad mediante el alfa de Cronbach. La metodología de análisis utilizada se realizó en tres secuencias: primera, un análisis preliminar; segunda, un análisis factorial exploratorio (AFE) y; tercera, un análisis factorial confirmatorio (AFC).

\section{RESULTADOS}

Para describir cuales son las condiciones de la gestión del talento humano que facilitan el desarrollo de las capacidades dinámicas se realizó un análisis preliminar de las encuestas por tres expertos. Una vez validada la encuesta, se procedió aplicarlas para verificar que los cuestionarios se han realizado de acuerdo a las instrucciones y que se han rellenado de forma apropiada. Asimismo, se revisó la codificación de las variables. La grabación y validación de los datos se hizo con los programas estadísticos SPSS y AMOS para posteriormente realizar un análisis factorial llevado a cabo en dos secuencias. En una primera secuencia se 
ha realizado un AFE a partir de la técnica de las componentes principales iteradas y rotación Varimax. El análisis de Káiser- Meyer- Olkin es de 0,860 y el alfa de Cronbach arrojó un valor de 0,961. Por tanto, la escala es fiable y existe intercorrelación entre las variables que la conforman. El contraste de Bartlett ( $\mathrm{x} 2$ $=383,527 ; \mathrm{gl}=28$ y $\mathrm{p}=0,00)$ rechaza la hipótesis nula de no correlación significativa entre las variables observadas. Por tanto, es adecuada la aplicación del análisis de componentes principales a las variables. La extracción de factores arroja un resultado de dos factores retenidos (con un auto valor superior a 1). El conjunto de estos explica el $69,890 \%$ de la varianza total de las variables observadas. El primer factor, capacidades dinámicas, se midió por medio de las variables relaciones con el entorno, conocimiento externo y oportunidades o necesidades del mercado. El segundo factor, gestión del conocimiento, se midió a través de las variables espacio compartido de conocimiento y estilo de liderazgo transformacional.

Posteriormente, se procedió a contrastar y re-especificar el modelo, mediante un análisis factorial confirmatorio (AFC), los dos factores anteriores. Se consideró oportuno realizar esta secuencia para superar las limitaciones de la técnica de componentes principales, y con ello contrastar y perfeccionar los nuevos factores extraídos. Se ha buscado afianzar la unidimensionalidad de los factores, la fiabilidad de cada uno de ellos, así como la validez convergente y discriminante. Realizado el AFC, una vez hechas las reespecificaciones correspondientes, se han identificado los dos factores ya señalados. En la secuencia del AFC, para la identificación de los factores se ha seguido el método de máxima verosimilitud (ML). Para cada factor se ha realizado un análisis de normalidad multivariante. El hecho de que las variables observadas no sean normales no ha impedido la utilización de este método, porque el programa estadístico AMOS, es capaz de invertir la matriz de varianzas-covarianzas aun en esta situación (Acosta, Longo-Samoza, \& Fischer, 2013). Para mayor comprensión se agrupan todos los resultados obtenidos de los dos factores y variables observadas que los conforman en la tabla 2 y 3 . Por otro lado, con relación a la variable gestión del talento humano, se estudió desde las condiciones facilitadoras para el desarrollo de las capacidades dinámicas, encontrando que estas son el espacio compartido de conocimiento y el estilo de dirección.

Tabla 2. Factor: Capacidades dinámicas

\begin{tabular}{|c|c|c|c|c|c|c|}
\hline Variable & $\begin{array}{c}\text { Carga } \\
\text { factorial }\end{array}$ & KMO & $\begin{array}{l}\text { Test de } \\
\text { Bartlett }\end{array}$ & $\begin{array}{c}\text { Alfa de } \\
\text { Cronbach }\end{array}$ & $\begin{array}{c}\text { Variable } \\
\text { total }\end{array}$ & $\begin{array}{c}\text { Hipótesis } \\
\text { contrastada }\end{array}$ \\
\hline $\begin{array}{l}\text { La empresa mantiene relaciones } \\
\text { efectivas con el entorno que le } \\
\text { permiten obtener conocimiento } \\
\text { externo. }\end{array}$ & 0,951 & \multirow{6}{*}{0,825} & \multirow{6}{*}{$\begin{array}{l}X^{2}=167 \\
, 419 \\
g l=15 \\
p=0,00\end{array}$} & \multirow{6}{*}{0,805} & \multirow{6}{*}{68,466} & \multirow{6}{*}{$\mathrm{H} 1$} \\
\hline $\begin{array}{l}\text { La empresa desarrolla nuevos } \\
\text { procesos, productos o servicios a } \\
\text { partir de sus relaciones con el } \\
\text { entorno. }\end{array}$ & 0,893 & & & & & \\
\hline $\begin{array}{l}\text { La empresa descubre nuevas } \\
\text { oportunidades de negocio mediante } \\
\text { las relaciones con el entorno. }\end{array}$ & 0,818 & & & & & \\
\hline $\begin{array}{l}\text { Los productos y servicios ofrecidos } \\
\text { por la empresa responden a las } \\
\text { necesidades del mercado. }\end{array}$ & 0,796 & & & & & \\
\hline $\begin{array}{l}\text { La empresa invierte en conocimiento } \\
\text { para realizar actividades muy } \\
\text { específicas. }\end{array}$ & 0,738 & & & & & \\
\hline $\begin{array}{l}\text { El conocimiento adquirido supone un } \\
\text { alto grado de novedad para la } \\
\text { empresa. }\end{array}$ & 0,835 & & & & & \\
\hline
\end{tabular}

Los resultados generales evidencian que las condiciones de la gestión humana que favorecen el desarrollo de las capacidades dinámicas dependen de los conocimientos generados y una adecuada adaptación al entorno de estas empresas. Es así como los resultados demostraron que en la medida que se desarrollan espacios para crear, desarrollar y transferir conocimiento, compartir experiencias e ideas que propician la generación de soluciones técnicas de alto valor, con un estilo de liderazgo transformacional que favorezca el cambio, el desarrollo profesional y el aprovechamiento del potencial de los miembros de la empresa, la organización responde o se adapta a los cambios propuestos por el entorno. Esta interpretación es consistente con la hipótesis 2 ya que de este modo se evidencia que las condiciones de la gestión del talento humano, espacio compartidos de conocimiento y estilo de dirección favorecen el desarrollo de capacidades dinámicas. 
Tabla 3. Factor: Gestión del talento humano.

\begin{tabular}{|c|c|c|c|c|c|c|}
\hline Variable & $\begin{array}{c}\text { Carga } \\
\text { factorial }\end{array}$ & $\mathrm{KMO}$ & $\begin{array}{l}\text { Test de } \\
\text { Bartlett } \\
\end{array}$ & Alfa & $\begin{array}{l}\text { Variabl } \\
\text { e total }\end{array}$ & $\begin{array}{c}\text { Hipótesis } \\
\text { contrastada }\end{array}$ \\
\hline $\begin{array}{l}\text { En la empresa se aprovecha todo el } \\
\text { potencial de sus directivos y } \\
\text { colaboradores. }\end{array}$ & 0,730 & 0,825 & $\begin{array}{c}X 2=387,527 \\
g \mid=28 \quad p=0,00\end{array}$ & 0,860 & 74,623 & $\mathrm{H} 2$ \\
\hline $\begin{array}{l}\text { Los integrantes de la empresa } \\
\text { conforman para compartir } \\
\text { experiencia y conocimiento. }\end{array}$ & 0,820 & \multirow{7}{*}{0,825} & \multirow{7}{*}{$\begin{array}{c}X^{2}=387,527 \\
g l=28 \\
p=0,00\end{array}$} & \multirow{7}{*}{0,860} & \multirow{7}{*}{74,623} & \multirow{7}{*}{$\mathrm{H} 2$} \\
\hline $\begin{array}{l}\text { Los cambios tecnológicos de la } \\
\text { empresa son asumidos con facilidad } \\
\text { por los colaboradores. }\end{array}$ & 0,745 & & & & & \\
\hline $\begin{array}{l}\text { Los empleados entregan de manera } \\
\text { libre y espontánea soluciones } \\
\text { técnicas de alto valor a problemas } \\
\text { de la organización. }\end{array}$ & 0,840 & & & & & \\
\hline $\begin{array}{l}\text { La empresa favorece el desarrollo } \\
\text { profesional de los empleados. }\end{array}$ & 0,791 & & & & & \\
\hline $\begin{array}{l}\text { La empresa cuenta con un programa } \\
\text { formalizado para la gestión del } \\
\text { talento humano. }\end{array}$ & 0,874 & & & & & \\
\hline $\begin{array}{l}\text { La empresa entrega incentivos } \\
\text { económicos a los empleados que } \\
\text { formulen, ejecuten y desarrollen } \\
\text { propuestas innovadoras que } \\
\text { respondan a condiciones del } \\
\text { entorno. }\end{array}$ & 0,812 & & & & & \\
\hline $\begin{array}{l}\text { El liderazgo de la empresa favorece } \\
\text { el cambio y la transformación de } \\
\text { manera activa. }\end{array}$ & 0,715 & & & & & \\
\hline
\end{tabular}

Es así como la organización gestiona su talento humano, en la medida que establece relaciones de cooperación tanto externa como interna que permitan compartir experiencias y conocimiento de manera libre y espontánea. Adicionalmente, la gestión del talento favorece el desarrollo de capacidades dinámicas a través de procesos dinámicos e integrales que permiten a la empresa adaptar la base de recursos y capacidades para afrontar los cambios del entorno y generar nuevas formas de generación de ventajas competitivas por medio del talento de sus empleados. Este argumento es consistente con la hipótesis 1.

\section{CONCLUSIONES}

De acuerdo a la revisión de la literatura y a los hallazgos encontrados, se concluye que para el desarrollo de capacidades dinámicas se requiere de la gestión del talento humano para responder a las condiciones del entorno. De tal manera que se aprovechen y reconozcan las oportunidades del medio, a partir del establecimiento relaciones efectivas con actores externos. En este sentido, la habilidad y motivación de los empleados juega un papel preponderante para el desarrollo de capacidades dinámicas, ya que estas requieren en gran medida de la conformación de equipos de trabajo y de un estilo de dirección transformacional. Por tanto, este proceso involucra la colaboración entre las personas y el medio, facilitando la capacidad de relacionamiento. En este sentido, la investigación confirmó las condiciones facilitadoras de la gestión del talento humano que favorecen su generación son el espacio de interacción de conocimiento entre los colaboradores y un estilo de liderazgo que promueva la transformación y el cambio.

\section{REFERENCIAS}

Acosta, J., Creación y desarrollo de capacidades tecnológicas: un modelo de análisis basado en el enfoque de conocimiento. Tesis Doctoral. Universidad Autónoma de Madrid (2010).

Acosta, J., y Fischer, A. Condiciones de la gestión del conocimiento, capacidad de innovación y resultados empresariales. Un modelo explicativo. Pensamiento y Gestión, № 35 (2013).

Aguilar, J., y Yepes, E. Gestión de capacidades dinámicas e innovación: Una aproximación conceptual. Revista de Ciencias de Administración 8(16), 1-15 (2006).

Augier, M., y Teece, D Dynamic Capabilities and Multinational Enterprise: Penrosean Insights and Omissions. Management International Review, vol. 47(2), 175-192 (2007). 
Barrios-Hernandez, K., y Olivero, E. La Innovación en Instituciones de Educación Superior Un Modelo Basado en Capacidades Dinámicas. Barranquilla: Universidad Simón Bolívar (2015).

Barrios, K., Olivero, E., y Acosta-Prado, J. Capacidad dinámica de innovación en instituciones de educación superior. Revista Espacios. Vol 38 (1), 24 (2017)

Barrios-Hernández, K., Olivero, E y otros veintitrés autores Gestión del conocimiento y capacidad de innovación. Modelos, Sistemas y Aplicaciones. Barranquilla: Universidad Simón Bolívar (2017).

Barney, J., Firm resources and Sustained Competitive Advantage. Journal of Management 17(1), 99-120. (1991)

Becerra, F., y Álvarez, C. El Talento Humano y la Innovación Empresarial en el Contexto de las Redes Empresariales: El Clúster de Prendas de Vestir en Caldas- Colombia. Estudios Gerenciales (27) 119, 209-225 (2011).

Bierly, P., y Chakrabarti, A. Generic Knowledge Strategies in the U.S. Pharmaceutical Industry. Strategic Management Journal, 17 (winter special issue), 123-135 (1996).

Bravo, E., y Herrera, L. Generación de capacidades dinámicas mediante la innovación organizacional: Un múltiple estudio de casos exploratorio. 3rd International Conference on Industrial Engineering and Industrial Management. BarcelonaTerrassa, September 2nd-4th (2009).

Calderon, G., Cuartas, G y Álvarez, C. Transformación organizacional y prácticas innovadoras de gestión humana. Innovar (19)35, 151-166 (2009).

Dávila, J., Capacidades organizacionales: dinámica por naturaleza. Cuadernos de Administración 26(47), 11-33. (2013).

Eisenhardt, K., y Martin, J. Dynamic Capabilities: What are they. Strategic Management Journal 21, 1105-1121 (2000).

Garzón, M., El Constructo capacidades dinámicas. Guanajuato, México: Ide@s CONCYTEG 8(99) (2013).

Grant, R., Prospering in dynamically-competitive environments:Organizational capability as knowledge integration. Organization Science Vol 7(4), 375-387 (1996).

Helfat, C., y Peteraf., M. The Dynamic Resource-Based View: Capability Lifecycles. Strategic Management Journal, vol. $24, n^{\circ} 10,997-1010$ (2003).

Hernández, A., Marulanda, C. y López, M. Análisis de capacidades de gestión del conocimiento para la competitividad de Pymes en Colombia. Información Tecnológica. Doi: 10.4067/S0718-07642014000200013. Vol 25, no. 2, pp. 111-122 (2014)

Hung, H. F., Kao, H.P., Chu, Y. An empirical study on knowledge integration, technology innovation and experimental practice. Expert Systems with Applications, vol. 35, no. 1-2., 177-186 (2008).

Lavie, D., Capability reconfiguration: An analysis of incumbent responses to technological change, Academy of Management Review, vol. 31, no. 1, pp. 153-174 (2006).

Laursen, K., The importance of sectoral differences in the application of complementary HRM practices for innovation performance. International Journal of the Economics of Business (9), 139-156 (2002).

Mejía, G., y Jaramillo, M. Formación del talento humano: factor estratégico para el desarrollo de la productividad y la. Revista Científica Guillermo de Ockham (4) 1, 43-81 (2006).

Mejía, A., Bravo, M. y Montoya, A. El factor del talento humano en las organizaciones. Ingenería Industrial. Vol. XXXIV (1), 2-11 (2013).

Nishida, K. An Inquiry into the Good. Abe, M., and Ives, C. (trans.) (1990) New Haven/London: Yale University. (1921)

Nonaka, I., y Takeuchi, H. The knowledge-creating company. Oxford: Oxford University Press (1995).

Penrose, E., The theory of the growth of the firm. New York: John Wiley (1959).

Shimizu, H. Ba-Principle: New Logic for the Real-time Emergence of Information, Holonics, 5 (1), 67-69. (1995)

Teece, D., Explicating dynamic capabilities: The nature and microfundations of (sustainable) enterprise performarmance. Strategic Management Journal 28, 1319-1350 (2007).

Teece, D., y Pisano, G. The Dynamic Capabilities of Firms: An Introduction. Industrial and Corporate Changevol. Vol 3(3), 537-556 (1994).

Teece, D., Dynamic Capabilities y Strategic Management. Organizing for Innovation an Growth. New York: Oxford University Press Inc (2009).

Venture, V., Análisis competitivo de la empresa: un enfoque estratégico. Civitas: Madrid (1994).

Wang, C., y Ahmed, P. Dynamic Capabilities: "A review and Research Agenda". Stretegic Management Journal, $31-51$ (2007).

Zahra, S., y George, G. Absorptive capacity: A review, reconceptualisation, and extension. Academy of Management Review 27(2), 185-203 (2002.) 